\title{
Consequences for LHC from cosmic ray experiments
}

\author{
A.A. Petrukhin \\ National Research Nuclear University MEPhI \\ Moscow, Russia \\ E-mail: AAPetrukhin@mephi.ru
}

LHC energy range $1-14 \mathrm{TeV}$ in collider collisions corresponds to cosmic ray energy region $10^{15}-10^{17} \mathrm{eV}$. But namely in this region some very unusual results were obtained in cosmic ray experiments. Analysis of these results obtained both in hadron and in muon components shows that they cannot be explained in frame of existing theories and models. New interaction model based on production of blobs of quark-gluon matter (QGM) with high orbital momentum allows describe all observed unusual events. It is important that all unusual events appear at energies higher than $10^{15} \mathrm{eV}$ in nuclei-nuclei interactions. Therefore it is necessary to search new physics in LHC experiments in nuclei-nuclei interactions, too.

LHC on the March - IHEP-LHC

20-22 November 2012

Institute for High Energy Physics, Protvino,Moscow region, Russia 


\section{Introduction}

Discovery of Higgs boson was the main task for LHC, and its investigations will be continued. Of course, investigations of known processes and phenomena in new energy region up to $14 \mathrm{TeV}$ will be continued, too. This part of future experiments is important but not so promising. Naturally, practically all physicists think and dream about new physics. Especially, since there are various theoretical models with new ideas: suppersymmetry, dark matter etc. But some probability exists that new physics is being observed in cosmic ray experiments at corresponding energies.

LHC energies $1-14 \mathrm{TeV}$ correspond to the interval $10^{15}-10^{17} \mathrm{eV}$ in laboratory system for p-p-interactions (for nuclei-nuclei-interactions this interval can be several times higher). But namely at these energies and above many interesting and sometimes unusual results were obtained in CR experiments. Of course, in cosmic ray investigations there are many drawbacks. As a rule, in experiments are unknown: type of particles, their energy and direction, total flux, place and time of interaction. Nevertheless, the obtained results are very total and possibly indicate at new physics existence.

\section{Some results of experiments in cosmic rays above $10^{15} \mathrm{eV}$}

Historically, the first "unusual" result was obtained 55 years ago. In the measured spectrum of all particles in extensive air showers the change of its slope was observed [1]. And though in this first paper both possible reasons of its appearance - change of hadron interaction and change of primary cosmic ray energy spectrum were considered - in further interpretations the second reason was dominating.

The second "unusual" phenomenon was the behavior of the ratio of muon number to electron number with increasing of EAS energy. This phenomenon also can be interpreted in two ways: change of interaction model and change of mass composition of primary cosmic rays. The last version was dominating, since it was in a good agreement with galactic model of cosmic ray origin.

Other observed "unusual" pecualirities of EAS development (some excess of so-called "young" and "old" showers, very large transverse momenta, etc.) are not so impressive and can be explained by large fluctuations in hadron interactions.

The next group of more serious unusual events was observed in the Pamir experiment [2]. Such phenomena as Halos, Centauros, Alignment, Penetrating cascades cannot be explained by existing theoretical models, in spite of numerous attempts to do this.

But crucial results were obtained recently in muon experiments with muon bundles investigations and muon energy spectrum above $100 \mathrm{TeV}$ measurements. In papers [3, 4] it was shown that the number of muon bundles with multiplicity about 100 particles and more cannot be described by existing theoretical models even for pure iron composition of primary cosmic rays. In NEVOD-DECOR experiment [5] with inclined EAS it was shown (Fig. 1) that the excess of muon bundles increases not only with multiplicity, but with increasing of zenith angle of EAS detection, that is with increasing of primary particle energy. At energies more than $10^{18}$ $\mathrm{eV}$ the excess is higher than predictions for pure iron primary flux. This result was confirmed in 
Auger experiment [6], in which muon bundle flux is two times higher than prediction for energy more than $10^{19} \mathrm{eV}$. Last measurements of muon energy spectrum above $100 \mathrm{TeV}[7,8]$ also showed serious deviations from normal energy spectrum (Fig. 2).
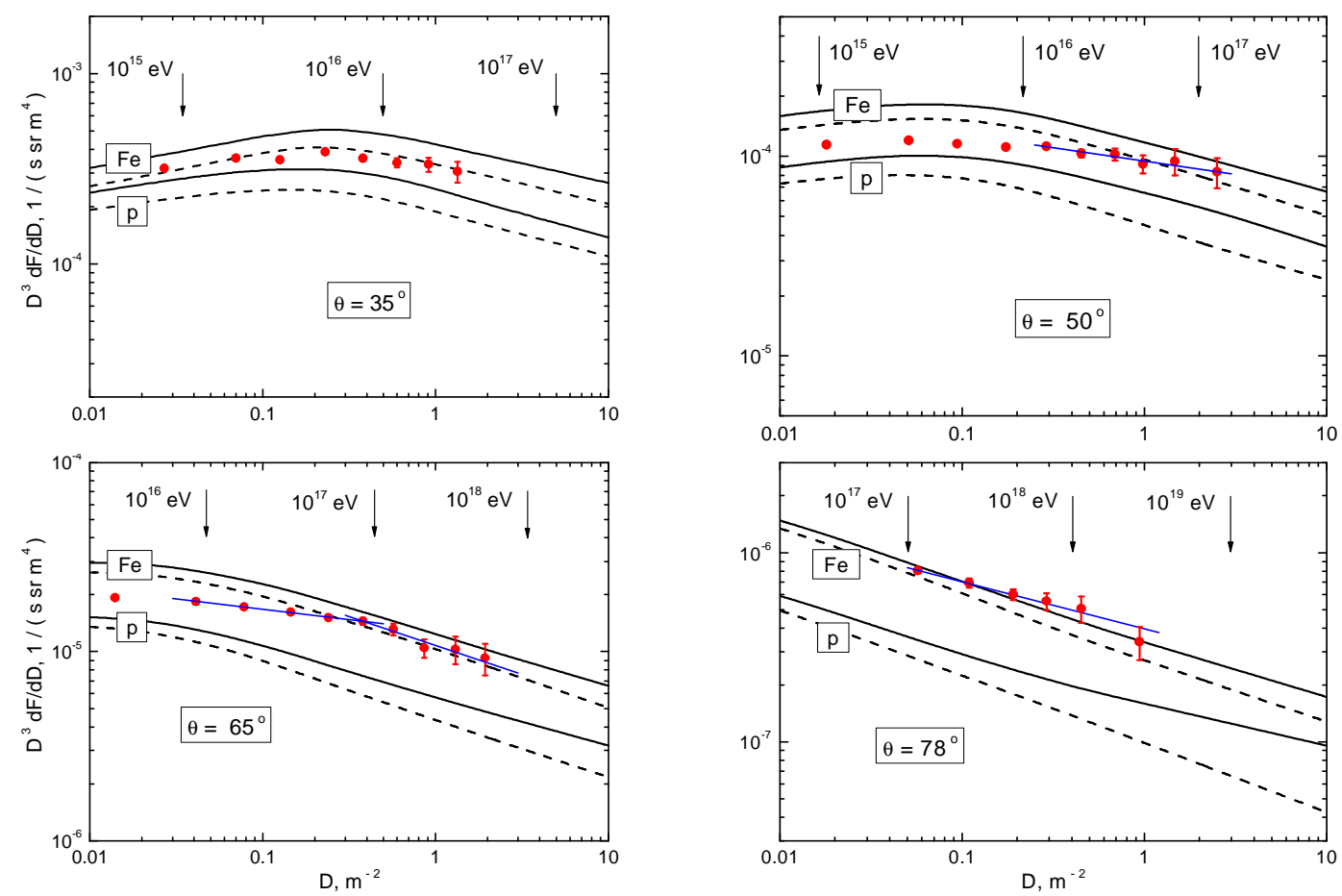

Figure 1: Experimental and calculated differential spectra of the local muon density at zenith angles of (a) $35^{\circ}$, (b) $50^{\circ}$, (c) $65^{\circ}$, and (d) $78^{\circ}$. Points represent experimental data; the solid and dashed curves correspond to results of calculations performed using the QGSJET01 and SIBYLL2.1 models, respectively. In each panel, the lower pair of curves corresponds to primary protons, while the upper pair corresponds to iron nuclei.

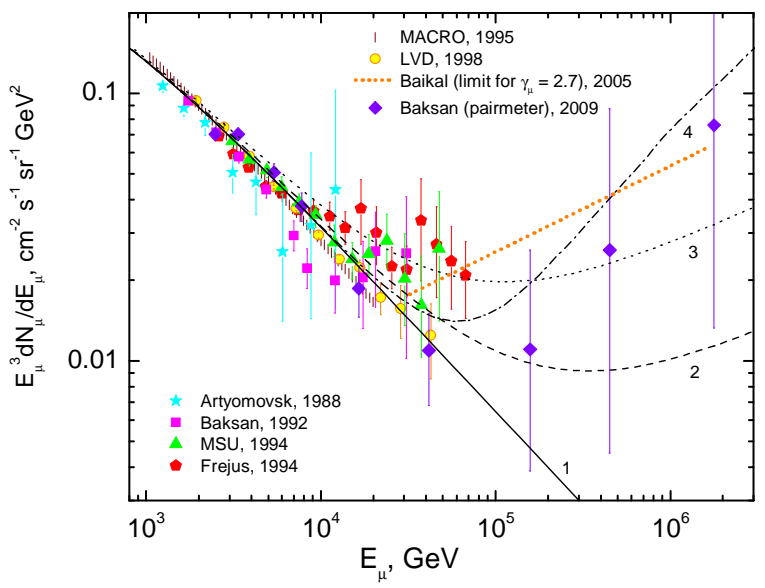

Figure 2: Differential muon energy spectra for vertical direction measured in various experiments (depth-intensity curve analysis and cascade shower spectrum measurements). The curves correspond to different spectrum models. Recent BUST results obtained by means of multiple interaction method are marked by solid diamonds [7]. 


\section{Possible explanation of unusual results in CR experiments}

In principle, each unusual result obtained in cosmic ray experiments, taking into account numerous uncertainties, can be explained in the frame of existing models as a tail of corresponding distribution. But it is impossible to explain the full set without introduction of a model change. Among of proposed ideas, sometimes very exotic ones appear. The most realistic is the model of production of blobs of quark-gluon matter (QGM) with large orbital momentum [9]. This model allows explain all unusual phenomena detected in cosmic ray experiments:

1. Threshold behavior of unusual events (they appear at several PeV only) since for QGM blob production a certain high temperature (energy) is required.

2. Large cross section which is necessary to change the slope of EAS spectrum and give possibility to observe various unusual events in cosmic rays is being obtained as result of the transition from quark (gluon)-quark (gluon) interaction to some collective interaction of many quarks and gluons:

$$
\sigma=\pi \lambda^{2} \rightarrow \pi(\lambda+R)^{2} \text { or } \pi\left(R_{1}+R_{2}\right)^{2}
$$

where $R, R_{1}$ and $R_{2}$ are sizes of quark-gluon blobs.

3. For explanation of such very different events as alignment, missing energy and excess of VHE muons and muon bundles a single phenomenon - large orbital momentum - is required. The appearance of large orbital momentum at non-central ionion collisions was discussed by two China theorists [10]. The value of this orbital momentum can reach $L \sim 10^{5}$ which is necessary for alignment explanation. On the other hand, the centrifugal barrier $V(L)=L^{2} / 2 m r^{2}$ appears, which will be high for the light quarks $(u$ and $d$ ), but low for heavy top-quarks. Production of top-quarks with a large cross section drastically changes the hadron interaction, since after a decay of $t$ quark into $b$-quark and $W$-boson, a consecutive decay of $W$-boson can give as leptons $(l$, $\left.v_{e}\right) \sim 30 \%$ so multiple $(\sim 20)$ pions $\sim 70 \%$. The first channel gives an excess of VHEmuons and missing energy (three neutrinos), and the second channel gives an excess of muon bundles due to anomalous increasing of pion multiplicity.

Such a change of interaction model allows explain the changes of primary cosmic ray energy spectrum and mass composition which are extracted from results of EAS investigation. Since for QGM blob production not only a high temperature but a high density of matter is required, in the first turn QGM blobs appear in interactions of heavy nuclei of cosmic rays (iron) with nuclei of air. This phenomenon can explain both EAS energy spectrum slope increase and the tendency to a heaver estimated mass composition.

\section{Some predictions for future LHC experiments}

Simulations based on CORSIKA with introducing of top-quarks using PYTHIA showed [11] that the best agreement with existing experimental data can be obtained if 
the threshold energy for different nuclei of primary cosmic rays depends on atomic number as

$$
E_{\text {th }}=E_{\text {knee }}\left(\frac{56}{A}\right)^{0.5} .
$$

In this model, for interaction of protons with nuclei of air the threshold energy is more than $10^{16} \mathrm{eV}$. Apparently, for p-p-interaction the threshold energy of QGM blob production will be more than $10^{17} \mathrm{eV}$ (more than $14 \mathrm{TeV}$ in the center-of-mass system for collider experiments). Therefore this phenomenon cannot be observed in ppinteractions at LHC and its investigations must be done in nuclei-nuclei interactions, starting with at least nitrogen-nitrogen collisions.

But all methods of the search of top-quarks are developed for p-p-interaction only. Therefore development of methods of the search of top-quarks in nuclei-nuclei collisions is required. Apparently, some evidences of new processes in nuclei-nuclei interactions in LHC experiments were observed. Among them: i) strong increase of charged particle multiplicity compared to results of calculations based on existing models [13]; ii) detection of highly asymmetric di-jet events [14], which can be explained in frame of QGM blob production model, if one takes into account that $\mathrm{t}$ quarks decay into b-quarks and W-bosons, which in their turn can decay into $\sim 20$ pions.

Possibly one of perspective methods of searching of new state of matter (QGM blobs with large orbital momentum) in LHC experiments will be investigations of missing energy, which must increase with increasing of colliding particle energy.

In any case it is quite possible that the first searches of new state of matter will be much easier in cosmic rays than in collider experiments.

\section{Conclusions}

If the considered approach to explanation of numerous unusual events detected in cosmic rays above $10^{15} \mathrm{eV}$ based on QGM blob production with large orbital momentum is correct, then this new state of matter can be found in LHC experiments in nuclei-nuclei interactions only.

\section{Acknowledgement}

This work has been supported by the Russian Ministry of Education and Science, RFBR (grant 11-02-1222-ofi-m-2011) and grant of the Leading Scientific School NSh-6817.2012.2. Author thanks Aleksey Bogdanov and Rostislav Kokoulin for the help in preparation of this paper. 


\section{References}

[1] G.B. Khristiansen and G.V. Kulikov, Zhurn. Experim. Teor. Fiz. (In Russian) 35 (1958) 635.

[2] S.A. Slavatinsky, Nucl. Phys. B (Proc. Suppl.) 122 (2003) 3.

[3] V. Avati et al., Astropart. Phys. 19 (2003) 513.

[4] J. Abdallah et al., Astropart. Phys. 28 (2007) 273.

[5] A.G. Bogdanov et al., Phys. Atom. Nucl. 73 (2010) 1852.

[6] G. Rodriguez, Proc. 32nd Int. Cosmic Ray Conf. Beijing, China, 2011. Vol. 2. P. 95.

[7] A.G. Bogdanov et al., Astropart. Phys. 36 (2012) 224.

[8] P. Berghaus, Atmospheric muon spectrum from catastrophic energy losses in IceCube. Talk presented at 32nd Int. Cosmic Ray Conf. (Beijing), 2011.

[9] A.A. Petrukhin, Proc. Vulcano Workshop "Frontier Objects in Astrophysics and Particle Physics”, 2006. P. 497. (Ital. Phys. Soc. 2007. Vol. 93).

[10]Zuo-Tang Liang and Xin-Nian Wang, Phys. Rev. Lett. 94 (2005) 102301.

[11]A.A. Petrukhin, Nucl. Instr. and Meth. A 692 (2012) 228.

[12]A.A. Petrukhin, Nucl. Phys. B (Proc. Suppl.) 212-213 (2011) 235.

[13]G. Tonelli, Results from the experiments at the LHC, Talk presented at 32nd Int. Cosmic Rays Conf., Beijing, 2011.

[14]G. Aad et al., Phys. Rev. Lett. 105 (2010) 252303. 